\title{
Analysis of Probabilistic Classification Learning in Patients With Parkinson's Disease Before and After Pallidotomy Surgery
}

\author{
Jennifer R. Sage, ${ }^{1,4,6}$ Stephan G. Anagnostaras, ${ }^{1,4,5}$ Shawn Mitchell, ${ }^{1}$ \\ Jeff M. Bronstein, ${ }^{2}$ Antonio De Salles, ${ }^{3}$ Donna Masterman, ${ }^{2}$ and Barbara J. Knowlton ${ }^{1}$ \\ Departments of ${ }^{1}$ Psychology, ${ }^{2}$ Neurology, and ${ }^{3}$ Neurosurgery, University of California, Los Angeles, California 90095, USA; ${ }^{4}$ Department of \\ Psychology, Emory University, Atlanta, Georgia 30322, USA; ${ }^{5}$ Center for Behavioral Neuroscience, Atlanta, Georgia 30322, USA
}

\begin{abstract}
This study examined the characteristics of probabilistic classification learning, a form of implicit learning previously shown to be impaired in patients with basal ganglia dysfunction (e.g., Parkinson's disease). In this task, subjects learn to predict the weather using associations that are formed gradually across many trials, because of the probabilistic nature of the cue-outcome relationships. Patients with Parkinson's disease, both before and after pallidotomy, and age-matched control subjects, exhibited evidence of probabilistic classification learning across 100 training trials. However, pallidotomy appears to hinder the learning of associations most implicit in nature (i.e., weakly associated cues). Although subjects were most sensitive to single-cue associations when learning the task, there is evidence that cue combinations contribute significantly to probability learning. The utility of multiple dependent measures is discussed.
\end{abstract}

Many studies have provided evidence for the existence of multiple memory systems in the brain (Mishkin et al. 1984; Packard et al. 1989; Packard and White 1991; Eichenbaum 1992; Packard and McGaugh 1992; Squire 1992; Kesner et al. 1993; McDonald and White 1993; Schacter and Tulving 1994; Salmon and Butters 1995; Squire and Knowlton 1995; Knowlton et al. 1996a; Packard and McGaugh 1996; Floresco et al. 1997). For example, the medial-temporal lobe (MTL) system (including the hippocampus) appears to mediate rapidly acquired "declarative" memory. In contrast, certain learning disrupted by neostriatal damage (including the dorsolateral caudate nucleus or nigrostriatal system) is mediated by a procedural memory system characterized by slowly acquired associations and often referred to as habit learning (Mishkin et al. 1984; Sage and Knowlton 2000).

Patients with basal ganglia dysfunction as a result of Parkinson's disease (PD) or Huntington's disease (HD) display impairments in perceptual motor-skill learning yet can achieve normal performance on several implicit memory measures. Implicit tasks with less of a motor component, such as artificial grammar and visuospatial category learning, have been shown to be intact in patients with PD or HD (Reber and Squire 1999; Knowlton et al. 1996b). However, one task with very little motor component has been reported to be impaired in these patients: the weather prediction probabilistic classification task. In this paradigm,

${ }^{6}$ Corresponding author.

E-MAIL: jsage@emory.edu; FAX: (404) 727-0372.

Article and publication are at http://www.learnmem.org/cgi/doi/ 10.1101/lm. 45903 . cue-outcome associations are learned gradually over many trials. Amnesic patients with hippocampus damage are unable to declaratively recollect even the basic appearance of the cues, while still exhibiting probabilistic classification learning comparable to controls (Knowlton et al. 1994, 1996a,b; Reber et al. 1996). In this "weather" task, the relationship between a set of shape cues and their weather outcome is probabilistic. Information gained from a single trial is not nearly as useful as information gained across several trials (one hallmark of habit learning). Subjects are told that they are a weather forecaster and their goal is to predict "sun" or "rain" given each cue or set of cues that appears on the screen, as pictured in Figure 2, below.

The second component of this task is a declarative memory questionnaire, which tests subjects' memory of certain aspects of the task, including the appearance of the screen. It is with these combined implicit and declarative memory measures that a double dissociation has been shown between amnesic patient performance and Parkinson's patient performance. That is, amnesics (with MTL damage) show normal habit learning and impaired declarative memory, while PD patients show impaired habit learning and normal declarative memory (Knowlton et al. 1996a). More recently, studies have sought to further define the circuit responsible for this form of habit learning. Evidence has emerged from fMRI studies demonstrating activation in the neostriatum during learning in the weather prediction task in normal subjects, and a decrease in activation in medial temporal lobe structures (Poldrack et al. 1999, 2001). Alzheimer's patients, with presumably intact

LEARNING \& MEMORY 10:226-236 @ 2003 by Cold Spring Harbor Laboratory Press ISSN1072-0502/03 \$5.00

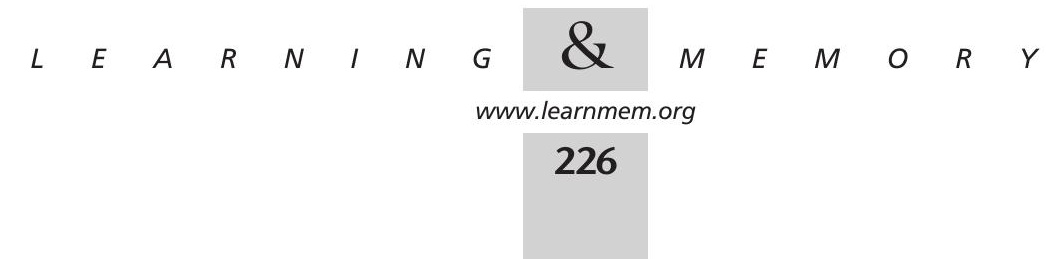




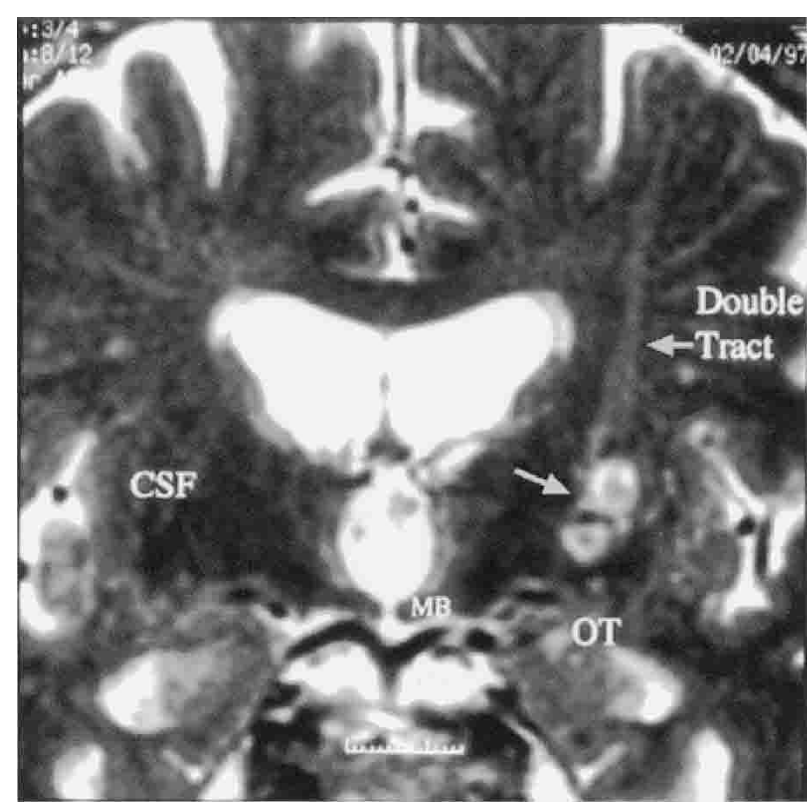

Figure 1 Coronal T2-weighted magnetic resonance imaging (MRI) scan showing a double electrode track for generation of a pallidotomy lesion away from the internal capsule and optic tract. Notice the mammillary body at the level of the pallidotomy lesion. Two lesions were performed to encompass the globus pallidus interna (GPI) (horizontal arrow). CSF, cerebral spinal fluid; $M B$, mammillary body; OT, optic tract.

subcortical structures, perform as well as controls on the weather prediction task (Eldridge et al. 2002).

However, characteristics of the learning acquired in the probabilistic classification task have not been examined fully. Although prior studies have assessed declarative memory for the weak and strong cue-outcome associations learned in the weather task (Reber et al. 1996), actual implicit performance on the task has not previously been dissociated in this way. To more fully characterize the properties of this form of implicit habit learning, the current study examined patterns of performance in normal elderly subjects, as well as in patients with PD both before and after pallidotomy surgery.

Pallidotomy is a brain lesioning procedure that, after years of refinement of technique and localization, experienced a resurgence for the treatment of the motor symptoms prevalent in advanced PD (Laitinen et al. 1992). Many patients with PD experience immediate and dramatic motor improvements after a lesion of part of their ventral posterior internal globus pallidus (Gpi; Fig. 1). This lesion is thought to be effective because it targets a sensorimotor area of the pallidum and attenuates the overactive inhibitory output of the GPi. Although pallidotomy is not a cure for PD, sustained motor improvements have been demonstrated months to years after surgery (Masterman et al. 1998).

PD patients were tested on the probabilistic classification task before and after pallidotomy surgery. Given the previously described deficit in probabilistic classification learning in patients with PD, we investigated whether this lesion procedure would improve both motor and implicit memory performance. Although neurosurgeons strategically target the motor portion of the GPi (see Masterman et al. 1998 for lesion placement in the current patients), the compact nature of the motor and cognitive mapping from the cortex onto the globus pallidus leaves open the possibility that several corticostriatal pathways, and not just the motor path, could be affected.

Recent studies examining other cognitive effects of pallidotomy have yielded mixed results. Many studies have found little or no major effects on cognition using standardized neuropsychological tests (Baron et al. 1996; Soukup et al. 1997; Fields et al. 1998; Masterman et al. 1998; Merello et al. 1999; Vingerhoets et al. 1999; Yokoyama et al. 1999). Others have found improvements in verbal memory (Manning et al. 1997), still others have found deficits in frontallobe-sensitive tasks (Lang et al. 1997; Trépanier et al. 1998; Stebbins et al. 2000; Rettig et al. 2000), and many describe problems with verbal fluency (Lang et al. 1997; Tröster et al. 1997 [stimulation]; Scott et al. 1998; Trépanier et al. 1998; Wilde et al. 1999). However, implicit memory abilities, including habit learning, have yet to be examined.

In this study, we describe characteristics of normal probabilistic classification learning in a group of control subjects and in patients with PD, pre- and postpallidotomy. Subjects with PD were tested within a few days before and an average of 3 months after surgery.

\section{RESULTS}

\section{Characteristics of Normal Probabilistic Classification Learning}

\section{Percent Correct Analyses}

Figure 3A depicts mean percent correct for normal elderly subjects performing the weather task. A MANOVA revealed significant improvement in probabilistic classification learning across the 100 trials, $[F(4,60)=5.74 ; P=.0006]$. These participants displayed performance not significantly above chance in the first trial block, with a subsequent increase in performance well above chance (Fig. 3A; one-sample t-test with hypothesized mean of $50 \%$ [chance]; for trial block 20 , $P>.05$; for trial blocks $40,60,80$ or $100, P<.01$ ].

These subjects also displayed normal declarative memory for the appearance and structure of the weather task, as assessed by a multiple-choice questionnaire (Fig. 3B). Subjects scored substantially above chance (25\%) on this 8 -item test $[T(15)=12.96 ; P<.0001]$.

\section{Cue Combination Analysis}

A more detailed analysis of the data was performed by examining responses to cue combinations that were strongly or weakly associated with particular weather outcomes. Of

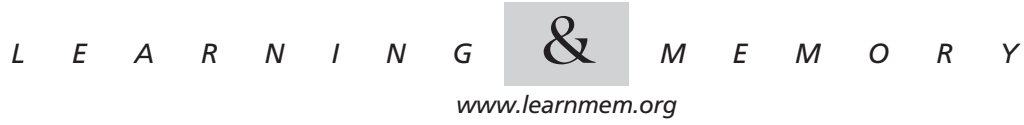



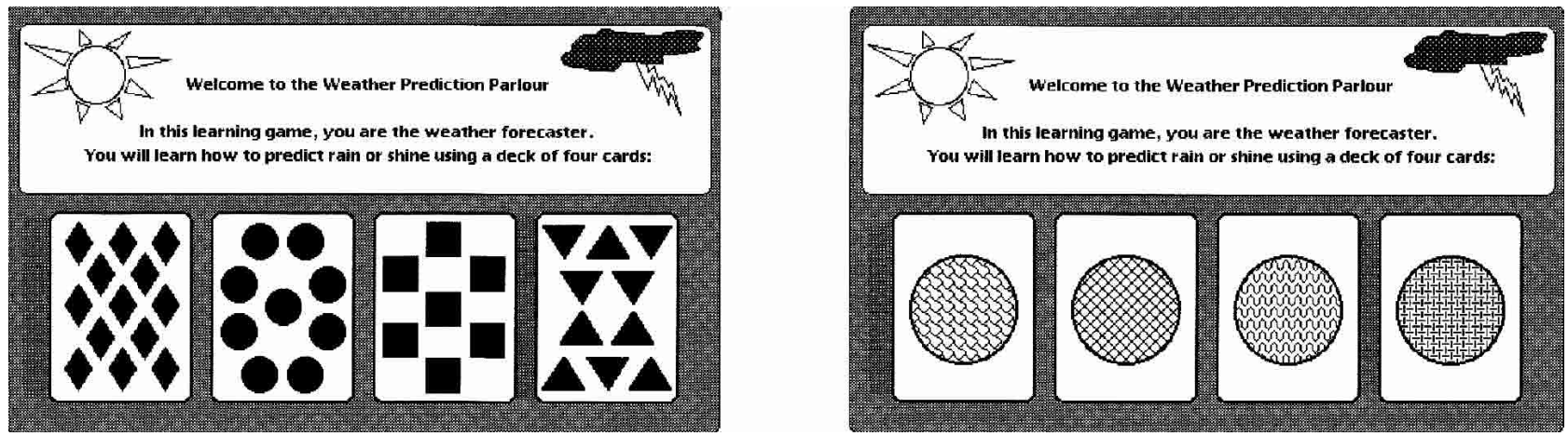

Figure 2 Cues used by subjects to predict the weather in a probabilistic manner (the probabilistic classification weather prediction task). One to three cards appeared on the screen during each trial. The subject was required to predict sun or rain based on these cues.

the 14 possible cue combinations, two possible combinations represented equal (and opposite) weather outcomes, and therefore were not included in this analysis. These will be referred to as "toss" trials.

The 12 meaningful cue combinations were grouped into weakly (63 and 67\%) and strongly $(75,83,86$, and $89 \%$ ) associated weather outcomes. Figure 4A illustrates subjects' choice accuracy for these combinations. Performance was above chance for strongly associated combinations $(T[15]=3.61 ; P=0.003)$, but not for weakly associated combinations $(T[15]=1.15 ; P=.266)$.
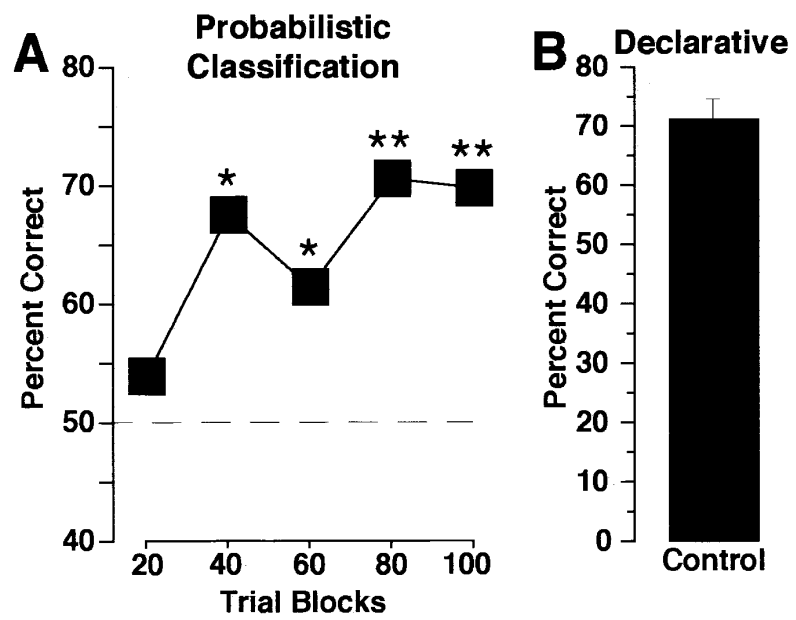

Figure 3 (A) Probabilistic classification. Performance of normal, elderly control subjects on the 100-trial probabilistic classification (weather prediction) task is depicted as percent correct (number correct/total number of responses) across 20 trial blocks. Asterisks indicate performance significantly different from chance (dashed line, $50 \%{ }^{*} P<.05,{ }^{*} P<.0005$; one-sample t-test). These subjects exhibited a typical learning pattern, starting at chance and increasing by the second block to well above chance performance. Responses were considered correct if they corresponded to the more strongly associated outcome (sun or rain) on each trial. (B) Declarative memory. Subjects performed well above chance $(25 \%)$ on an eight-item, four-choice questionnaire (shown as number correct/8), which assessed the appearance and characteristics of the task.

\section{Cue Combination Probability Matching}

In addition to comparing how well subjects performed on weakly and strongly associated cue combinations, we examined control subjects' probability matching performance for the cues presented. This analysis differs from above, in that it does not rely upon a percent correct score. Whereas the percent correct measure assesses the subjects' abilities to respond with the most strongly associated outcome for a given set of cues, Figures $4 \mathrm{~B}$ and 4C illustrate subjects' abilities to match the programmed probabilities of each combination of cues or individual cues.

Figure $4 \mathrm{~B}$ displays the programmed strengths of each cue combination ("Actual") and subjects' accuracy in matching those strengths across the 100 training trials. The associative strengths of the cue combinations presented were $89 \%, 86 \%, 83 \%, 75 \%, 67 \%$, and $63 \%$. The dependent measure, Outcome Response Probability, is defined as the probability of the most associated response (either rain or sun) when the pattern was present. As depicted in Figure $4 \mathrm{~B}$, subjects significantly discriminated among the six combinations $(F[5,75]=3.84 ; P=.004)$, and there was a modest linear correlation ( $r=0.24 ; P=.017$; Fisher's r-to-z) between the programmed cue combination strengths and subjects' response probabilities. Performance was closer to the actual probabilities for the single cues (.86 and .63) compared to the patterns with multiple cues.

\section{Single Cue Strength Analysis}

In addition to analyzing cue combination probability matching, we explored subjects' accuracy in matching the programmed probabilities of the single cues presented. Were subjects more closely matching cue combinations or single cue strengths?

Figure $4 \mathrm{C}$ displays the programmed strengths of each cue stimuli ("Actual") and subjects' accuracy in matching those strengths. Outcome response probability was defined as the probability of a "rain" response whenever the cue stimulus was present (alone or in combination with other cues). Subjects significantly differentiated between the four 


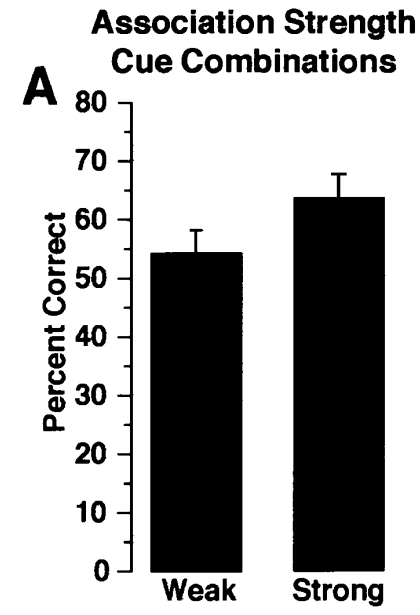

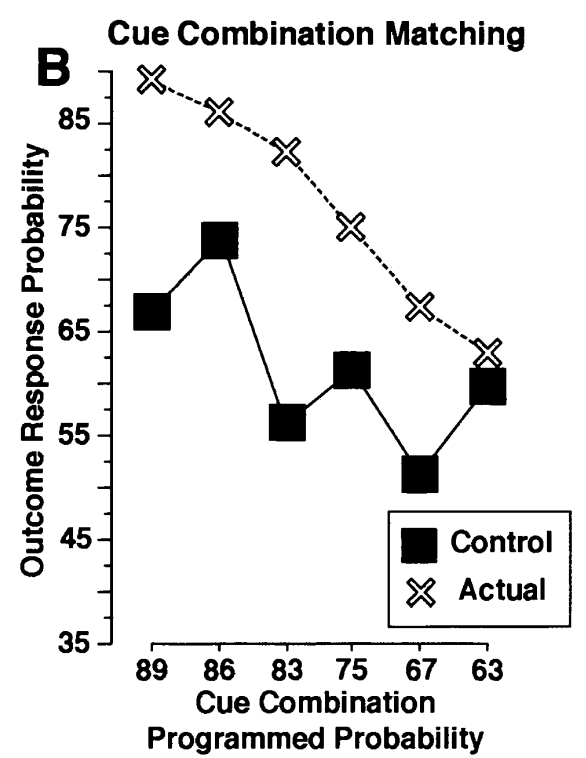

Figure 4 (A) Association strength-cue combinations. Control subjects exhibited above chance accuracy when using strongly associated cue combinations $(75,83,86$, and $89 \%$ ), but not weakly associated combinations (63 and $67 \%$ ). (B) Cue combination probability matching. The probability of a response in the presence of each cue combination (squares) and the actual programmed probability (Xs). Subjects significantly matched combination strengths but not to a very strong degree. (C) Individual cue probability matching. The probability of a response in the presence of each of the four individual cues compared with programmed probability, irrespective of whether cues appeared in a combination or alone. Normal subjects matched individual cue probabilities to a greater degree than combinations.

stimuli probabilities, $(F[3,45]=17.24 ; P<0.001)$. Importantly, there was a strong linear correlation $(r=0.712$; $P<.0001)$ between the programmed single cue strengths and subjects' response probabilities.

Overall, the programmed individual cue strengths (Fig. 4C) were much better predictors of subject performance than the cue combination strengths (Fig. 4A,B).

\section{Probabilistic Classification Learning in Subjects With PD Before and After Pallidotomy Surgery} Throughout this section, results will be given for both between-groups (All Patients) and within-subjects (Retest) analyses. All patients analyses include patients tested either before or after pallidotomy, plus subjects tested both before and after pallidotomy $(n=17)$. Retest analyses refer to the latter group of subjects (tested at two time points $(n=7)$.

\section{Percent Correct Analyses}

Figure 5A depicts accuracy on the probabilistic classification task (All Patients, left; Retest patients, right). Examination of Figure 5A suggests that before pallidotomy ("preop"), patients with PD exhibited an atypical pattern of performance, starting with an unusually accurate first block of trials, declining to chance, and then recovering. Eight of the 12 subjects who were tested preoperatively performed above $60 \%$ during the first block of 20 trials. However, only four of these maintained this level of performance in the second block, and none of the other patients in this group scored above $60 \%$ on this block. In contrast, control subjects began closer to chance, as expected, and continued to improve to above-chance performance (Fig. 3A). Only four of the 16 control subjects performed above $60 \%$ in the first block of 20 trials. All four of these subjects continued to perform above this level in the next block of trials, and eight additional control subjects achieved this level in the second block.

The All Patients analysis (Fig. 5A, left) revealed no significant effects (MANOVA; group by trial interaction, $F[4,84]=1.36, \quad P=.255 ; \quad$ main effect of group, $F[1,21]=1.65, P=.213$; main effect of trial, $F[1,21]=1.89$, $P=.121$ ). Retest patients (Fig. 5B, right), however, exhibited significant change in performance across the 100 trials, and showed no significant deleterious effects of the surgery (main effect of trial, $F[1,6]=3.47, P=.023$; group by trial interaction, $F[4,24]<1, P>.70$; main effect of group, $F[1,6]<1, P=.50)$.

In contrast to their varied performance on the habitlearning task, all groups performed similarly well on the declarative memory questionnaire. Figure 5B illustrates no significant group differences in mean percent correct on the 8-item task, for both All Patients $(F[1,22]<1)$ and Retest $(F[1,6]<1)$ comparisons.

To more precisely analyze subjects' accuracies compared to chance, we utilized a one-sample t-test with a hypothesized mean of $50 \%$ (chance performance). Figures $3 \mathrm{~A}$

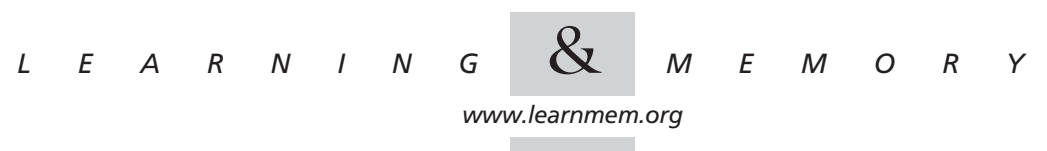




\section{Probabilistic Classification}

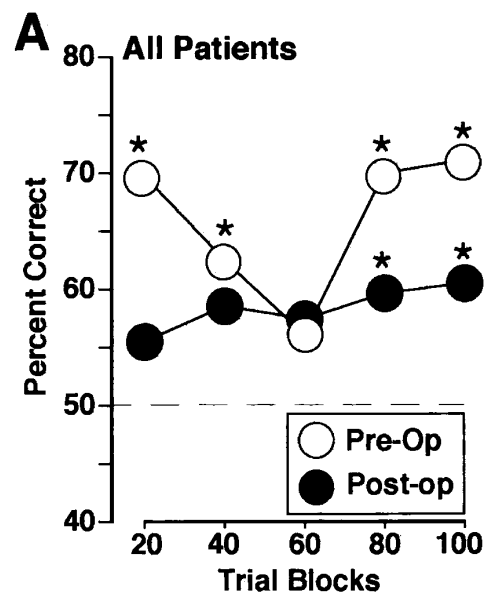

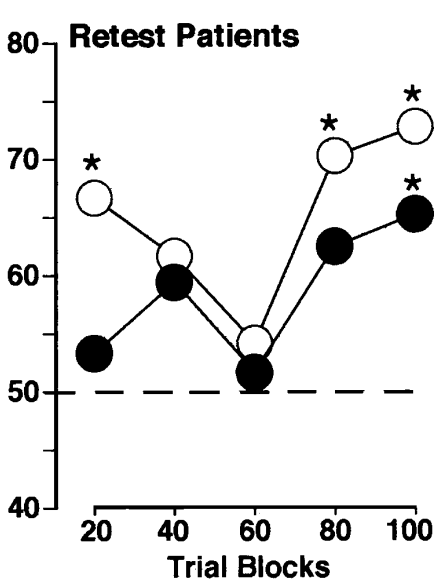

\section{Declarative}

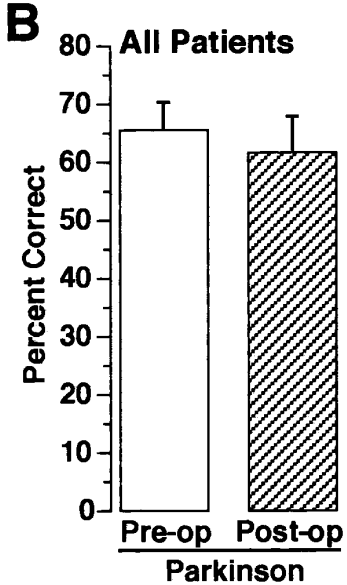

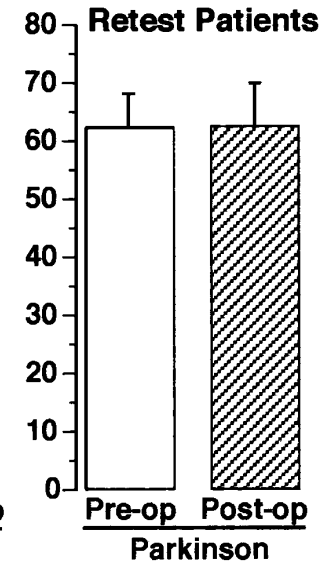

Figure 5 Performance of patients with Parkinson's disease before or after pallidotomy. (A) Probabilistic classification. Accuracy in 20 trial blocks is shown in patients that were tested before and/or after pallidotomy (All Patients, left panel), and a subset that were tested both before and after pallidotomy (Retest patients, right panel). Asterisks indicate performance significantly different from chance $(50 \%$; $* P<.05)$. Postoperative patients began at chance and did not exceed chance performance until the last block of trials. In contrast, control subjects (Fig. 3A) exceeded chance by the second block of trials. Preoperative patients appear to spuriously exceed chance on the first block of trials, decline to chance levels, and then exceed chance by the last block of trials. This performance is not as robust as control subjects, and may be the result of motivational factors. $(B)$ Declarative memory. Performance on an eight-item, four-choice declarative questionnaire regarding the weather prediction task is shown. All patients performed well above chance and comparable to control subjects (Fig. 3B) on this declarative memory test.

(controls) and 5A (patients) depict the group mean percent correct for each 20 trial block, and the results significantly above chance $\left({ }^{*} P<.05\right.$, $\left.{ }^{* *} P<.0005\right)$. Control subjects exhibited a typical learning pattern, significantly exceeding chance performance by 40 trials, and remaining above chance for each trial block thereafter. In contrast, preoperative subjects performed unusually well in the first trial blocks, followed by several trials at chance performance, and finally exceeded chance levels for the last two trial blocks. These qualitative results suggest that patients with PD perform abnormally on this task. Postoperative subjects did not exceed chance performance until the final one or two trial blocks. Unlike controls, they did not exhibit significant improvement within the first 60 trials. Although the preoperative patients performed unusually well in the first block of trials, this level of performance was not maintained. It is possible that motivational factors selectively influenced the preoperative subjects (see Discussion).

To more fully examine this unusual pattern of preoperative performance, percent correct for the first instance and the last instance of each of the 12 meaningful cue combinations was averaged across the 100 trials for each subject (Fig. 6). (Of the 14 cue combinations, two combinations were "toss trials"; see above.) It was expected that performance would be near chance (50\%) for the first encounter of a cue combination. In contrast, performance should be significantly improved upon the last instance of the cue combination.
As expected, control accuracy did not differ from chance upon the first instance of a cue combination $(T[15]=0.98 ; P=.345)$, but was significantly above chance on the last instance of a cue combination $(T[15]=3.43$; $P=.004)$. For All Patients, preoperative subjects surprisingly performed significantly above chance on the first instance of a cue combination $(T[11]=2.84 ; P=.016)$ and remained significantly above chance for the last instance $(T[11]=2.63 ; P=.024)$. No other Parkinson's group performed significantly above chance at either the first or last instance ( $P$ s $>.134$; including Retest subjects, data not shown). The unusually high accuracy solely noted in the preoperative patients' performance, upon their very first encounter of a cue combination, suggests that these particular patients may have used a different strategy early in the task, including explicit memorization of individual cue associations and responded to new patterns based on this knowledge.

For certain cue combinations, the first occurrence may have occurred as late as trial 40 , and the last instance may have been as early as trial 60 . Therefore, we examined minimum and maximum scores without assuming each individual would necessarily score lowest and highest in the first and last 10-trial block. Each individual's maximum and minimum 10-trial block score for the first four blocks (early, trials 0-40) and last four blocks (late, trials 60-100) were compared by group (data not shown). For minimum and maximum early scores, and minimum late scores, there

$$
\begin{array}{lllllllllllllll} 
& E & A & R & N & I & N & G & \mathcal{Q} & M & E & M & O & R & Y \\
\text { www.learnmem.org } & & &
\end{array}
$$




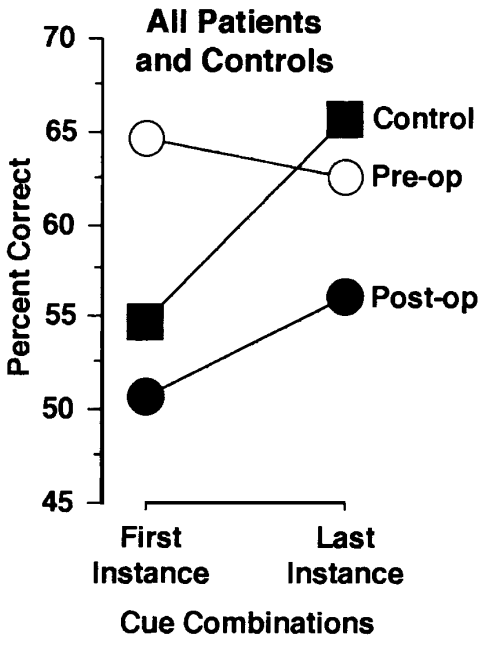

Figure 6 Performance on the first and last instance of each cue combination. To determine if the unusually high accuracy by preoperative patients in the first block of trials was spurious (Fig. 5A), we examined accuracy on the first and last instance of each cue combination. Control and postoperative patients began near chance, whereas preoperative patients were significantly above chance. Control subjects show a clear pattern of improvement, whereas both patient groups with Parkinson's disease show no evidence of improvement.

were no group differences (univariate ANOVAs, $F[2,36]$ values $<1.5, P$ values $>.2$ ]. However, there were significant group differences for the maximum percent correct score achieved during late trials $(F[2,36]=3.56 ; P=.04)$. Postoperative patents did significantly worse than control $(P=.04$; Fisher's PLSD) or preoperative patients $(P<.02)$. Thus, the absolute maximum performance exhibited by postoperative subjects is significantly worse than other groups.

\section{Cue Combination Analysis}

Because the lesions did not target cognitive circuitry, and because patients with PD have previously been shown to perform poorly on the habit-learning task, we did not expect a large effect of pallidotomy on this task. Because the above accuracy deficits are relatively subtle, we hypothesized that differences may be detected by separately examining weakly and strongly associated outcomes.

Figure 7A depicts the All Patients analysis of choice accuracy (expressed as percent correct) for weakly and strongly associated cue patterns. For weakly associated outcomes, postoperative patients were impaired relative to both preoperative patients $(F[1,21]=5.63 ; P=.027)$, and controls $(F[1,25]=4.59 ; P=.042)$, which did not differ from one another. There were no significant group differences in predicting strongly associated outcomes $(F[1,21]<1 ; P>.50)$. Retest data (not plotted) were similar (weakly associated: $F[1,6]=4.70 ; P=.073$; strongly associated: $F[1,6]<1 ; P>.50)$.
Importantly, postoperative patients who were tested both before and after pallidotomy did not display interference effects as a result of multiple testing, as they did not differ in performance from postoperative patients tested only once (after pallidotomy) (weak cue combinations, $F[1,9]<1 ; P>.50 ;$ strong cue combinations, $F[1,9]<1$; $P>.50)$.

\section{Cue Combination Probability Matching}

Figure $7 \mathrm{~B}$ depicts significant discrimination among the six cue combination strengths (All Patients: $F[5,110]=4.11$; $P=.002$; Retest (not plotted): $F[5,30]=4.53 ; P=.003$ ). While preoperative subject responses did not significantly correlate with the programmed cue combinations (All Patients, $r=.20 ; P=.095$ ), postoperative subject responses did modestly correlate (All Patients, $r=0.33 ; P=.004$ ). The preoperative patients appeared to perform relatively closer to actual probabilities for single cues compared to patterns with multiple cues. However, the postoperative patients performed particularly poorly on the weakly associated single cue (actual probability, .63).

\section{Single Cue Strength Analysis}

Figure $7 \mathrm{C}$ represents the programmed strengths of each cue, and subjects' accuracy in matching those strengths across the 100 training trials. Subjects significantly discriminated between the four stimuli probabilities (All Patients, $F[3,66]=17.4 ; P<.0001)$, but showed no significant group differences $(\mathrm{F}[1,22]<1 ; P>.3)$. Retest subjects performed similarly (data not plotted; main effect of cue, $F[3,18]=$ $7.93 ; P=.001 ;$ main effect of group, $F[1,6]=2.28$; $P=.182)$. Similar to normal elderly subjects, there was a strong linear correlation between the programmed single cue strengths and patients' response probabilities (All Patients preoperative: $r=0.7169 ; P<.0001$; All Patients postoperative: $r=0.572 ; P<.0001$; Fisher's r-to-z). Similar to control performance, the programmed individual cue strengths (Fig. 7C) were much better predictors of patient performance than cue combination strengths (Fig. 7B).

The appearance of Figure $7 \mathrm{C}$ suggests that postoperative patients had particular trouble in using more ambiguous cues $(57.5 \%, 42.5 \%)$, as compared to stronger cues $(75.6 \%, 24.4 \%)$. Therefore, we examined the partial correlations between programmed probability and patient response probability for strong versus weak individual cue strengths. For strong cues, all groups' performance correlated well with the programmed probabilities (control, $r=0.75 ; P<.0001$; preoperative, $r=0.75 ; P<.0001$; postoperative, $r=0.70 ; P<.0001)$. For weak cues, control $(r=0.54 ; P=.001)$, and preoperative subjects' $(r=0.62$; $P<.001)$ performance correlated well with the programmed probability. However, postoperative performance was uncorrelated for these weak cues $(r=-0.19 ; P>.3)$. Whereas control subjects and prepallidotomy patients are

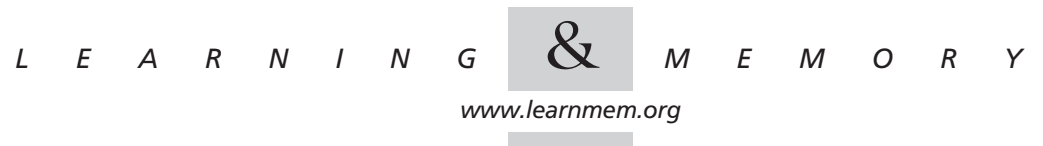



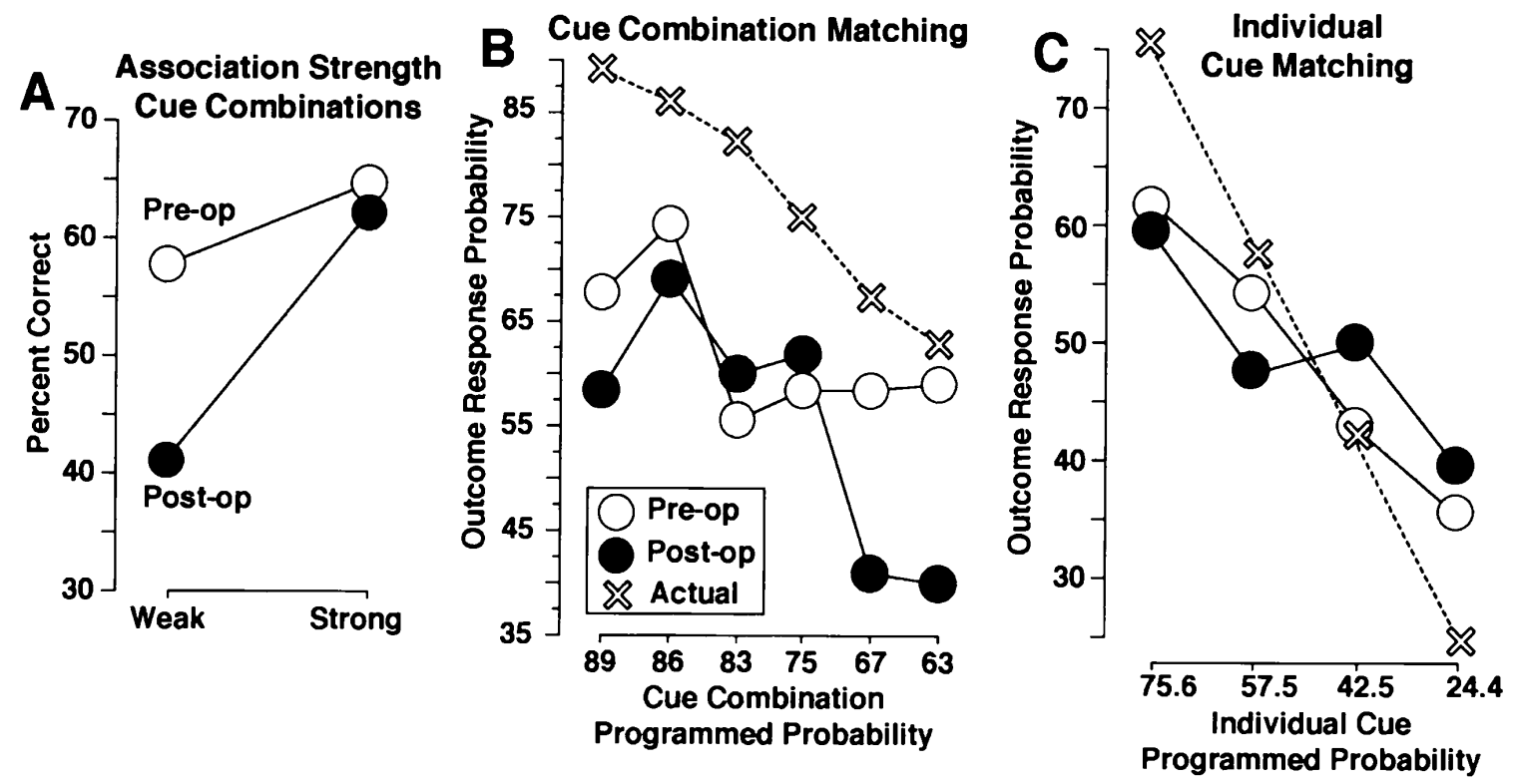

Figure 7 (A) Association strength—cue combinations. Postoperative patients with Parkinson's disease exhibited similar accuracy to preoperative patients when using strongly associated cue combinations $(75,83,86$, and $89 \%)$, but were somewhat impaired when using weakly associated combinations (63 and 67\%). Compare with control subjects (Fig. 4A). (B) Cue combination probability matching. The probability of a response in the presence of each cue combination (squares) with the actual programmed probability (Xs). Subjects significantly matched combination strength but not to a very strong degree; moreover, probability matching was somewhat worse in postoperative patients. Compare with control subjects (Fig. 4B). (C) Individual cue probability matching. The probability of a response in the presence of each of the four individual cues compared with programmed probability, irrespective of whether cues appeared in a combination or alone.

surprisingly accurate at matching the programmed probabilities for these more ambiguous cues (57.5\%, 42.5\%), pallidotomy appears to impair this ability.

\section{DISCUSSION}

The current study sought to specify characteristics of normal probabilistic classification learning in a group of elderly subjects matched to pre- and postpallidotomy patients with PD. Elderly subjects with no diagnosed neurologic condition showed significant learning across 100 trials of the weather task, as assessed by a percent correct measure. As expected, these subjects also exhibited intact declarative memory for the appearance of the task. Detailed examination of performance on various trial types revealed that performance is derived more from abstracting individual cue strengths than using cue combination associations. It should be noted, however, that these strategies are not orthogonal: Individual cue performance measures include trials when the cue is presented alone or in combination with other cues. In addition, cue combinations are, by definition, made up of individual cues. Therefore, these measures have some degree of overlap. It is important to note that in this implicit memory task, subjects are not solely using individual cues (cues perhaps most amenable to declarative memorization) to perform the weather task. Subjects also display significant probability matching for cue combinations.
The current results additionally suggest that pallidotomy has a subtle effect on probabilistic classification learning in patients with PD, which is most apparent for the weakly associated cues. Performance was similar in those patients who were retested after pallidotomy, and those tested for the first time after pallidotomy, suggesting that the findings cannot be attributed to negative transfer across test sessions. The patients with PD displayed qualitatively different patterns of performance on the weather task both before and after pallidotomy surgery, relative to controls. As reported in prior studies, declarative memory of the task was intact in all subjects.

The results from the weather prediction task were generally consistent with a prior study of the performance of PD patients on this task (Knowlton et al. 1996a). In that study, PD patients showed no learning across the first 50 trials with performance rising above chance only during the second 50 trials. In this study, neither preoperative nor postoperative patients sustained improvement in performance across the first 50-60 trials, but both groups did exhibit above-chance performance in later blocks. However, the preoperative patients actually performed above chance in the first two blocks and then dropped to nearchance levels. This result is puzzling, and it is difficult to discern whether it is spurious or represents the results of a different strategy used by PD patients in this task. This pattern was not seen in postoperative patients or control sub-

$$
\begin{array}{lllllllllllllll} 
& E & A & R & N & I & N & G & \mathcal{Q} & M & E & M & O & R & Y \\
\text { www.learnmem.org } & & &
\end{array}
$$


jects and differs from the earlier study with PD patients, suggesting that it is not a consistent finding. One possibility is that the preoperative patients were significantly motivated to perform well on the task. These patients were tested at the University of California, Los Angeles (UCLA) Medical Center in their physician's office immediately following final motor and cognitive testing by their physicians to ensure candidacy for pallidotomy. Although all subjects were assured their performance on the weather task would not affect their medical treatment, it is possible that they were more inclined to apply increased concentration, attention, or effort. These variables would not have affected the postoperative patients who had already received the surgery, nor the control subjects.

Although the influence of motivational factors on probabilistic classification performance has not been formally examined, Eldridge et al. (2002) found that control subjects who were informed they were participating in a study of memory as a comparison group for patients with Alzheimer's disease initially performed better on the weather task than a group of controls to which no mention was made of Alzheimer's disease or memory. These authors discussed the possibility that the "informed" control subjects may have assumed a strategy of explicit memorization of cues that may have improved their performance early in the task, but decreased it later, as cue associations intermittently reversed and negative feedback was provided. In this respect, the preoperative $\mathrm{PD}$ patients may have made choices based on simple heuristics derived from a few cue outcome associations encountered in the earliest trials, with this strategy abandoned as the task proceeded. Differing from prior studies (Knowlton et al. 1996a), the current study used verbal responses in order to reduce mistrials resulting from poor motor ability in subjects with PD. If the verbal responses were easier for the PD patients, this may have freed resources to allow some memorization of cues during early trials. In addition, the verbal response may have encouraged a declarative strategy. However, it is unclear why control subjects do not adopt this strategy, or why pallidotomy surgery should abolish this strategy. Further studies with PD patients using the weather task are needed to determine whether this early above-chance performance is replicable.

When the results were broken down by trial type, it appeared that after pallidotomy, patients performed more poorly on trials in which cue patterns were more weakly associated with an outcome. Postpallidotomy patients were actually numerically below chance on these patterns, perhaps attributable to choosing the opposite outcome for the similar pattern previously encountered. It is hypothesized that weakly associated trials may be most characteristic of habit learning, as explicit memory for individual trials is less helpful. However, the groups did not differ on performance for the strongly associated cues. Similar to control perfor- mance, individual cue strengths were much better predictors of patient performance than cue combination strengths.

The above results suggest that the standard plotting of the percent correct accuracy measure, used to score performance on the weather task, may not be ideal. As the structure of the task is designed to provide for gradual learning of associations across large numbers of trials (habit learning), the dissection of accuracy into blocks of 10 or 20 trials (especially early in training) may not result in a meaningful measure of learning. More meaningful is a measure that incorporates larger number of trials, and thereby increased exposure to the cues and cue combinations to be learned. In this study, the average last "first appearance" of a cue combination was on trial 39 (out of 100). Thus, depending upon the particular probability structure of the task, caution should be used when referring to accuracy results within the first several trial blocks, as subjects may not yet have encountered every cue combination to be learned. Because a cue combination must be presented twice in order to ascertain learning, it would seem that examination of larger trial blocks would provide more accurate results.

Control subjects with no known neurologic condition and subjects with neostriatal damage (PD) do use a probability matching strategy when performing the weather task, but are most sensitive to single cue associations. Therefore, caution should be also used when choosing the programmed strengths of the cues and combinations. Although spacing the programmed probabilities so that each cue or combination outcome is more distinct may increase accuracy, one potential result of this spread would be to make the cues more amenable to a declarative knowledge of the outcome. If the goal is to encourage an implicit learning strategy (Knowlton et al. 1994), then it is important to include probabilities less readily explicit.

The current findings are consistent with other studies demonstrating mild cognitive impairments following pallidotomy for PD (Hariz and De Salles 1997; Manning et al. 1997; Tröster et al. 1997; Masterman et al. 1998; Scott et al. 1998; Stebbins et al. 2000; Wilde et al. 1999; Rettig et al. 2000). However, caution should be used when generalizing these findings, as there is evidence that variations in exact lesion location (between patients and between surgical centers) provide different cognitive results (Lombardi et al. 2000). In addition, neuropsychological results may differ depending upon postsurgical testing interval (Baron et al. 1996; Trépanier et al. 1998; Yokoyama et al. 1999; Rettig et al. 2000). Furthermore, studies have shown that pallidotomy can change the effects of dopamine agonists on neuropsychological function (Alegret et al. 2000). While all of the current subjects with PD were tested "on" medication for this study, performance pre-and postpallidotomy may have differed if subjects had been tested "off" medication. 
In summary, pallidotomy surgery for PD appears to result in a mild exacerbation of implicit learning deficits measured by the probabilistic classification task, although this is outweighed by the beneficial effects on clinical measures of functional capacity (Masterman et al. 1998). Additionally, it is apparent from the analyses of trial types that care should be taken when manipulating the probability structure of the weather task or when choosing an appropriate dependent measure of implicit learning. Postpallidotomy subjects exhibit selective deficits in learning about cues that are more ambiguous, more weakly predictive, while normal elderly subjects and prepallidotomy PD patients demonstrate intact performance on these same cues. Specific cognitive deficits in implicit learning may be identified by further examination of these types of cues.

\section{MATERIALS AND METHODS}

\section{Subjects \\ Non-Parkinson's Control Subjects}

Sixteen subjects ( 8 males, 8 females) age- and education-matched to a group of patients with PD were tested in this study. These subjects were recruited through the Los Angeles community, had agreed to participate via a consent form approved by the UCLA Institutional Review Board, and were paid $\$ 10$ for their participation. These subjects verbally confirmed a normal neurologic history (i.e., no diagnosed neurologic disorder). They averaged $61 \pm 1.7$ $($ mean \pm S.E.M. $)$ years of age (range $=52-72)$ with $14.9 \pm 0.5$ years of education (range $=12-20$ ), and were tested once.

\section{Patients With Parkinson's Disease}

Seventeen patients with PD, referred through the UCLA Movement Disorders Clinic, were tested in this study: Five patients were tested preoperatively only (four males; one female), five postoperatively only (three males, two females), and seven additional patients were tested both pre- and postoperatively (referred to as "Retest"; six males, one female). Preoperative patients were tested 1-3 days before pallidotomy surgery and 3 to 6 months postsurgery, immediately following a scheduled neurological exam. Motor disability was assessed by the attending neurologist (D.M.), using the Unified Parkinson's Disease Rating Scale (UPDRS; Fahn and Elton 1987), Hoehn and Yahr (H\&Y) staging (Hoehn and Yahr 1967), and the Schwab and England Functional Assessment Scale (1969). The mean H\&Y stage ("on" medication) was $2.4 \pm 0.3$ for all preoperative patients (range $=1-3$ ), and $2.6 \pm 0.4$ for 12 of the 13 postoperative patients (range $=1-4$ ), for which data was available (where $1=$ least severe and $5=$ most severe). The mean H\&Y stages for patients tested at two time points (Retest) were as follows: $2.7 \pm 0.3$ preoperative (range $=1-3$ ) and $2.7 \pm 0.6$ for six of the seven postoperative subjects (range $=0-4$ ). The Activities of Daily Living (ADL) subscore of the UPDRS assesses patient functionality (where $52=$ most impaired and $0=$ normal functionality). Pallidotomy patients have been shown to exhibit improvement in ADL subscores following surgery (Masterman et al. 1998). The available data from the patients in the present study is consistent with a small improvement in ADL scores postsurgery. The mean ADL ("on medication") was $18.7 \pm 2.0$ for 6 of the 12 preoperative patients for which data was available (range $=13-26$ ), and $16.7 \pm 2.2$ for 11 of the 12 postoperative patients (range $=1-30$ ), for which data was available. The mean ADL for patients tested at two time points were: $19.4 \pm 2.3$ for five of the seven preoperative subjects (range $=13-26$ ) and $17.3 \pm 2.8$ for six of the seven postoperative subjects (range $=11-30$ ). The average age of the groups was $63 \pm 1.8$ years for preoperative patients and $65 \pm 2.0$ years for postoperative patients (total range $=52-73$ ). Preoperative patients averaged $14 \pm 0.7$ years of education, while postoperatives averaged $15 \pm 0.8$ years (total range $=12-20$ ). Of the 14 patients for which medication information was available, all were receiving dopamine precursor treatment (Carbidopa/Levodopa), and were tested while on medication. Four patients were also taking a monoamine oxidase inhibitor (Eldepryl), six were taking a dopamine-enhancing drug (Amantadine, Symmetrel, or Permax), one was taking a serotonin agonist (Serzone), and five were taking anticholinergic medication (Pro-Banthine, Artane, or Ditropan). Because these anticholinergics are given preferentially to younger patients with no signs of cognitive impairment, it is impossible to assess these medication effects on performance in the current study independently of these other factors.

Patients in this study were deemed suitable candidates for pallidotomy surgery (per their attending neurologist), and as such, had been previously screened for overt signs of dementia (according to the criteria of the DSM-IV), severe declarative memory deficits (using the 10-word shopping list [McCarthy et al. 1981] among other measures), and for other degenerative or structural brain abnormalities. Finally, one patient was excluded from our results as a result of highly elevated Beck (Beck et al. 1961) and Neuropsychiatric Inventory (Cummings et al. 1994) depression scores.

The target was localized based on magnetic resonance imaging (MRI) visualization of the GPi, as well as the relationship of the target to structures, such as the optic tract, internal capsule, and mammillary body (De Salles and Hariz 1998). The coordinates dictated by Laitinen et al. (1992) were used as starting point for target determination. Precise localization of the target was achieved by microelectrode recordings and electrical stimulation to elicit responses in the internal capsule, optic tract, and GPi itself (Lim and De Salles 1997). A MRI of a representative pallidotomy is shown in Figure 1.

\section{Probabilistic Classification Task (Implicit Learning)}

The probabilistic classification task was presented using a Macintosh PowerBook laptop computer. The structure of the task was similar to that used by Knowlton et al. (1996a). Four different cues (Fig. 2) were each independently associated with one of two outcomes (sun or rain). Each trial consisted of a presentation of one, two, or three of these cues. In all, there were 14 possible cue combinations, which were randomized across 100 trials with the constraint that the same cue combination could not appear twice in a row, and each outcome could not appear more than five successive times. The two outcomes occurred equally often across the 100 training trials. Each individual cue occurred $\sim 68 \%, 46 \%, 34 \%$, or $22 \%$ with a particular weather outcome. Thus, when the outcome was sun, cue 1 occurred on $68 \%$ of the trials, cue 2 occurred on $46 \%$ of the trials, cue 3 on $34 \%$ of the trials, and cue 4 on $22 \%$ of the trials. When the outcome was rain, cue 1 occurred on $22 \%$ of the trials, cue 2 on $34 \%$ of the trials, cue 3 on $46 \%$ of the trials, and cue 4 on $68 \%$ of the trials. Across all patterns, cues 1 and 4 occurred 45 times each and cues 2 and 3 occurred 40 times each during the 100 trials. For each subject, the four cues were randomly assigned to positions $1-4$.

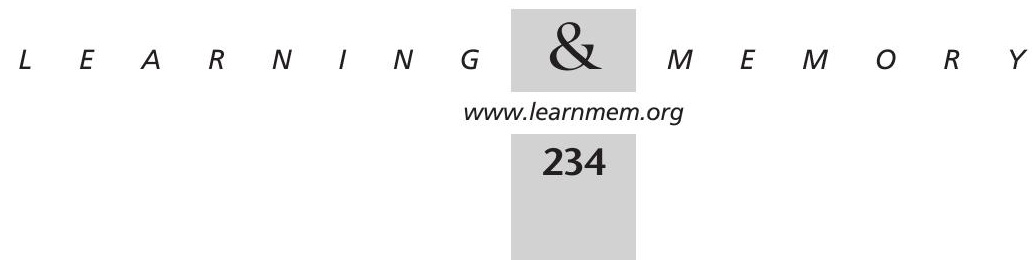


As some patients were tested both pre- and post-operatively, two sets of cues were used (Fig. 2): (1) geometric shapes, and (2) patterned circles. The cue set given pre- and postoperatively was counterbalanced across subjects. The use of the geometric cues has been described in prior patient studies (Knowlton et al. 1994, $1996 a, b)$. Selection of the second set of cue shapes (patterned circles) was determined by a pilot study using UCLA undergraduates to determine that both sets of cues produced comparable performance (data not shown).

\section{Multiple Choice Declarative Questionnaire}

Following completion of the computer task, each subject was administered an eight-item, multiple-choice questionnaire designed to assess explicit memory for the testing episode. Sample questions included, "How many squares were on the card with squares?" and "What appeared above the cards when you made a correct response?" Based on previous work (Knowlton et al. 1996a) and on the site of the lesion, we predicted that patients and controls would show similar performance on this declarative questionnaire.

\section{Procedure}

All patient testing occurred at the UCLA Medical Center, and control subject testing occurred at the subject's residence or at the UCLA Cognitive Neuroscience Laboratory. Subjects were informed they were participating in a weather-prediction task, with the two outcomes being rain or shine. On each trial, between one and three cue cards were presented and the subject was instructed to predict rain or shine based on the total set of cues that appeared. Subjects were told that although they may be guessing the outcome initially, performance would likely improve gradually.

Patients with PD often have difficulty initiating movements. As the weather prediction task requires subjects to respond quickly to each set of cues, the current study removed the manual motor component of the subject response, thereby minimizing mistrials (i.e., trials without a response). Both PD and control subjects indicated their prediction verbally and the experimenter then pressed the appropriate computer key, within $5 \mathrm{sec}$ of the cue presentation. If the subject did not respond within $5 \mathrm{sec}$, a prompt appeared on the screen, and the subject was asked to guess the outcome within the next $2 \mathrm{sec}$. Pilot studies in our laboratory have shown that patients with PD are readily able to make verbal responses during this time frame, yet it is short enough to discourage explicit memorization of the cue-outcome relationships. After each response, the computer delivered reinforcement according to the subject's response. Correct responses were followed by a pleasant tone and a smiling face, while incorrect responses were followed by a low tone and an unhappy face. The correct outcome (either a sun or a rain cloud) also appeared above the cues after the subject responded, and remained on the screen for $2 \mathrm{sec}$ before the next trial began. Subjects completed two blocks of 50 training trials separated by a short break (typically $<1 \mathrm{~min}$ ). Upon completion of the probabilistic classification task, the computer was turned off and subjects completed the multiple choice explicit memory questionnaire.

\section{Data Analysis}

For the percent correct scores, responses were considered correct if the subject selected the outcome that was most associated with the cue pattern. Because of the probabilistic nature of the task, cue combinations were sometimes followed by weakly associated outcomes (e.g., if cue 1 predicts sun $75.6 \%$ of the time, then $24.4 \%$ of the time it will predict rain). With this scoring system, subjects made a correct response by selecting the more strongly associated outcome (in this example, "sun"), even though that subject subsequently received feedback that an incorrect response was made. In this manner, the percent correct score more accurately reflected the learned-cue outcome association. The two cue combinations in which two cards predicted equally strong but opposite outcomes were excluded from the analysis, as the subject's response did not provide any information about classification learning.

Data were entered into a general multivariate normal analysis of variance (MANOVA); following a significant omnibus comparison, appropriate multiple posthoc comparisons were made, as described. Unless otherwise indicated, group by measure interactions were not significant and are not reported.

\section{ACKNOWLEDGMENTS}

This research was supported by a University of California, Los Angeles (UCLA) Academic Senate Grant to B.J.K. J.R.S. was supported by an individual National Institute of Mental Health National Research Service Award (NRSA) MH 12247, by an institutional NRSA MH 11061, and by a Will Rogers Fellowship. Portions of this research were presented at the Cognitive Neuroscience Society Annual Meeting, 1997. We thank Scott Mitchell and Michael Bassett for excellent subject recruitment and testing assistance, and Michael Fanselow, David Glanzman, and Carlos Grijalva for their thoughtful comments on an earlier version of this manuscript.

The publication costs of this article were defrayed in part by payment of page charges. This article must therefore be hereby marked "advertisement" in accordance with 18 USC section 1734 solely to indicate this fact.

\section{REFERENCES}

Alegret, M., Vendrell, P., Junque, C., Valldeoriola, F., Nobbe, F.A., Rumia, J., and Tolosa, E. 2000. Effects of unilateral posteroventral pallidotomy on 'on-off' cognitive fluctuations in Parkinson's disease. Neuropsychologia 38: 628-633

Baron, M.S., Vitek, J.L., Bakay, R.A.E., Green, J., Kaneoke, Y., Hashimoto, T., Turner, R.S., Woodard, J.L., Cole, S.A., McDonald, W.M., et al. 1996. Treatment of advanced Parkinson's disease by posterior GPi pallidotomy: 1-year results of a pilot study. Ann. Neurol. 40: 355-366.

Beck, A.T., Ward, Ch.H., Mendelson, M., Mock, J., and Erbaugh, J.K. 1961. An inventory for measuring depression. Arch. Gen. Psychiatry 4: 561-571.

Cummings, J.L., Mega, M., Gray, K., Rosenberg-Thompson, S., Carusi, D.A. and Gornbein, J. 1994. The neuropsychiatric inventory: Comprehensive assessment of psychopathology in dementia. Neurology 44: 2308-2314.

De Salles, A.A.F. and Hariz, M.I. 1998. MRI guided pallidotomy. In Neurosurgical operative atlas. (ed. S.S. Rengachary), Vol. 7, pp. 141-148. Williams \& Wilkins, Baltimore, MD. AANS

Eichenbaum, H. 1992. The hippocampal system and declarative memory in animals. J. Cogn. Neurosci. 4: 217-231.

Eldridge, L.L., Masterman, D., and Knowlton, B.J. 2002. Intact implicit habit learning in Alzheimer's disease. Behav. Neurosci. 116: 722-726.

Fahn, S. and Elton, R.L. 1987. Committee a.m.o.t. U.D., unified Parkinson's disease rating scale. In: Recent developments in Parkinson's disease. (eds. S. Fahn, et al.). Vol. 2. pp. 153-163. Macmillan Publishing Co. Inc., Florham Park, NJ

Fields, J.A., Tröster, A.I., Wilkinson, S.B., Lyons, K.E., Miyawaki, E., Pahwa, R., and Koller, W.C. 1998. Comparison of the cognitive safety of unilateral pallidal stimulation and pallidotomy. Neurology 52: A389.

Floresco, S.B., Seamans, J.K., and Phillips, A.G. 1997. Selective roles for

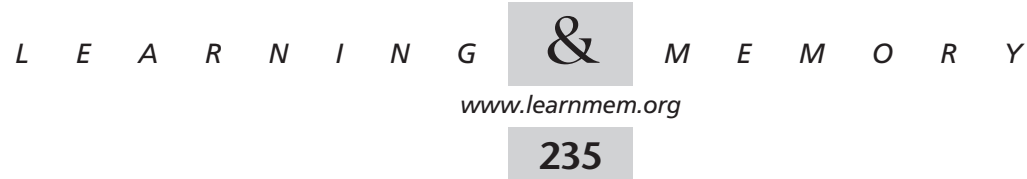


hippocampal, prefrontal cortical, and ventral striatal circuits in radial-arm maze tasks with or without a delay. J. Neurosci. 17: $1880-1890$.

Harriz, M.I. and DeSalles, A.A.F. 1997. The side-effects and complications of posteroventral pallidotomy. Acta Neurochir. 68: 42-48.

Hoehn, M.M. and Yahr, M.D. 1967. Parkinsonism: Onset, progression and mortality. Neurology 17: 427-432

Kesner, R.P., Bolland, B.L., and Dakis, M. 1993. Memory for spatial locations, motor responses, and objects: Triple dissociation among the hippocampus, caudate nucleus, and extrastriate visual cortex. Exp. Brain Res. 93: 462-470.

Knowlton, B.J., Squire, L.R., and Gluck, M.A. 1994. Probabilistic classification learning in amnesia. Learn. Mem. 1: 106-120.

Knowlton, B.J., Mangels, J.A., and Squire, L.R. 1996a. A neostriatal habit learning system in humans. Science 273: 1399-1402.

Knowlton, B.J., Squire, L.R., Paulsen, J.S., Swerdlow, N.R., Swenson, M., and Butters, N. 1996b. Dissociations within nondeclarative memory in Huntington's disease. Neuropsychology 10:538-548.

Laitinen, L.V., Bergenheim, A.T., and Hariz, M.I. 1992. Leksell's posterovental pallidotomy in the treatment of Parkinson's disease. $J$. Neurosurg. 76: 53-61.

Lang, A.E., Lozano, A., Tasker, R., Duff, J., Saint-Cyr, J., and Trépanier, L. 1997. Neuropsychological and behavioral changes and weight gain after medial pallidotomy. Ann. Neurol. 41: 834-835.

Lim, J. and De Salles, A.A.F. 1997. Long-term aspect of pallidotomy lesions on magnetic resonance. Stereotact. Funct. Neurosurg. 69: 46-53.

Lombardi, W.J., Gross, R.E., Trépanier, L.L., Lang, A.E., Lozano, A.M., and Saint-Cyr, J.A. 2000. Relationship of lesion location to cognitive outcome following microelectrode-guided pallidotomy for Parkinson's disease. Support for the existence of cognitive circuits in the human pallidum. Brain 123: 746-758.

Manning, C.A., Bennett, J.P., Wilkniss, S.M., Jones, M.G., and Laws, E.R. 1997. Comprehensive neuropsychological assessment of cognitive functioning pre- and post-unilateral posteroventral pallidotomy Neurology 48: A252.

Masterman, D., De Salles, A., Baloh, R.W., Frysinger, R., Foti, D., Behnke, E., Cabatan-Awang, C., Hoetzel, A., Intemann, P.M., Fairbanks, L., et al. 1998. Motor, cognitive, and behavioral performance following unilateral ventroposterior pallidotomy for Parkinson disease. Arch. Neurol. 55: 1201-1208.

McCarthy, M., Ferris, S.H., Clark, E., and Crook, T. 1981. Acquisition and retention of categorized material normal aging and senile dementia. Exp. Aging Res. 7: 127 -135.

McDonald, R.J. and White, N.M. 1993. A triple dissociation of memory systems: Hippocampus, amygdala, and dorsal striatum. Behav. Neurosci. 107: 3-22.

Merello, M., Nouzeilles, M.I., Kuzis, G., Cammarota, A., Sabe, L., Betti, O., Starkstein, S., and Leiguarda, R. 1999. Unilateral radiofrequency lesion versus electrostimulation of posteroventral pallidum: A prospective randomized comparison. Mov. Disord. 14: 50-56.

Mishkin, M., Malamut, B., and Bachevalier, J. 1984. Memories and habits: Two neural systems. In Neurobiology of learning and memory. (eds. G. Lynch, et al.). pp. 65-77. Guilford Press, New York, NY.

Packard, M.G. and McGaugh, J.L. 1992. Double dissociation of fornix and caudate nucleus lesions on acquisition of two water maze tasks: Further evidence for multiple memory systems. Behav. Neurosci. 106: 439-446.

Packard, M.G. and McGaugh, J.L. 1996. Inactivation of hippocampus or caudate nucleus with lidocaine differentially affects expression of place and response learning. Neurobiol. Learn. Mem. 65: 65-72.

Packard, M.G. and White, N.M. 1991. Dissociation of hippocampus and caudate nucleus memory systems by posttraining intracerebral injection of dopamine agonists. Behav. Neurosci. 105: 295-306.

Packard, M.G., Hirsh, R., and White, N.M. 1989. Differential effects of fornix and caudate nucleus lesions on two radial maze tasks: Evidence for multiple memory systems. J. Neurosci. 9: 1465-1472.
Poldrack, R.A., Prabhakaran, V., Seger, C.A., and Gabrieli, J.D. 1999. Striatal activation during acquisition of a cognitive skill. Neuropsychology 13: 564-574

Poldrack, R.A., Clark, J., Pare-Blagoev, E.J., Shohamy, D., Creso Moyano, J. Myers, C., and Gluck, M.A. 2001. Interactive memory systems in the brain. Nature 414: 546-550.

Reber, P.J. and Squire, L.R. 1999. Intact learning of artificial grammars and intact category learning by patients with Parkinson's disease. Behav. Neurosci. 113: 235-242.

Reber, P.J., Knowlton, B.J., and Squire, L.R. 1996. Dissociable properties of memory systems: Differences in the flexibility of declarative and nondeclarative knowledge. Behav. Neurosci. 110: 861-871.

Rettig G.M., Lai, E.C., Jankovic, J., Krauss, J.K., and Grossman, R.G. 2000. Neuropsychological outcome after unilateral pallidotomy for the treatment of Parkinson's disease. J. Neurol. Neurosurg. Psychiatry 69: 326-336.

Sage, J.R. and Knowlton, B.J. 2000. Effects of US devaluation on win-stay and win-shift radial maze performance in rats. Behav. Neurosci. 114: 295-306.

Salmon, D.P. and Butters, N. 1995. Neurobiology of skill and habit learning. Curr. Opin. Neurobiol. 5: 184-190.

Schacter, D.L. and Tulving, E. 1994. Memory systems 1994. MIT Press. Cambridge, MA.

Schwab, R.S. and England, A.C. 1969. Projection technique for evaluating surgery in Parkinson's disease. In Third symposium on Parkinson's disease. (eds., F.J. Gillingham and I.M.L. Donaldson), pp. 152-157. E\&S Livingstone, Edinburgh, Scotland

Scott, R., Gregory, R., Hines, N., Carroll, C., Hyman, N., Papanasstasiou, V., Leather, C., Rowe, J., Silburn, P., and Aziz, T. 1998. Neuropsychological, neurological and functional outcome following pallidotomy for Parkinson's disease: A consecutive series of eight simultaneous bilateral and twelve unilateral procedures. Brain 121: 659-675.

Soukup, V.M., Ingram, F., Schiess, M.C., Bonnen, J.G., Nauta, H.J.W., and Calverley, J.R. 1997. Cognitive sequelae of unilateral posteroventral pallidotomy. Arch. Neurol. 54: 947-950.

Squire, L.R. 1992. Memory and the hippocampus: A synthesis from findings with rats, monkeys, and humans. Psychol. Rev. 99: 195-231.

Squire, L.R. and Knowlton, B.J. 1995. Memory, hippocampus, and brain systems. In The cognitive neurosciences. (ed. M. Gazzaniga), pp 825-837. MIT Press, Cambridge, MA.

Stebbins, G.T., Gabrieli, J.D., Shannon, K.M., Penn, R.D., and Goetz, C.G. 2000. Impaired fronto-striatal cognitive functioning following posteroventral pallidotomy in advanced Parkinson's disease. Brain Cogn. 42: 348-363

Trépanier, L.L., Saint-Cyr, J.A., Lozano, A.M., and Lang, A.E. 1998. Neuropsychological consequences of posteroventral pallidotomy for the treatment of Parkinson's disease. Neurology 51: 207-215.

Tröster, A.I., Fields, J.A., Wilkinson, S.B., Pahwa, R., Miyawaki, E., Lyons, K.E., and Koller, W.C. 1997. Unilateral pallidal stimulation for Parkinson's disease: Neurobehavioral functioning before and 3 months after electrode implantation. Neurology 49: 1064-1083.

Vingerhoets, G., van der Linden, C., Lannoo, E., Vandewalle, V., Caemaert J., Wolters, M., and Van den Abbeele, D. 1999. Cognitive outcome after unilateral pallidal stimulation in Parkinson's disease. J. Neurol. Neurosurg. Psychiatry 66: 297-304.

Wilde, M.C., Plenger, P., Brookshire, B., and Van Horn, G. 1999 Neuropsychological outcome after unilateral posteroventral pallidotomy. Neurology 52: A232.

Yokoyama, T., Imamura, Y., Sugiyama, K., Nishizawa, S., Yokota, N., Ohta, S., and Uemura, K. 1999. Prefrontal dysfunction following unilateral posteroventral pallidotomy for Parkinson's disease. J. Neurosurg. 90: $1005-1010$

Received September 23, 2002; accepted in revised form February 13 , 2003.

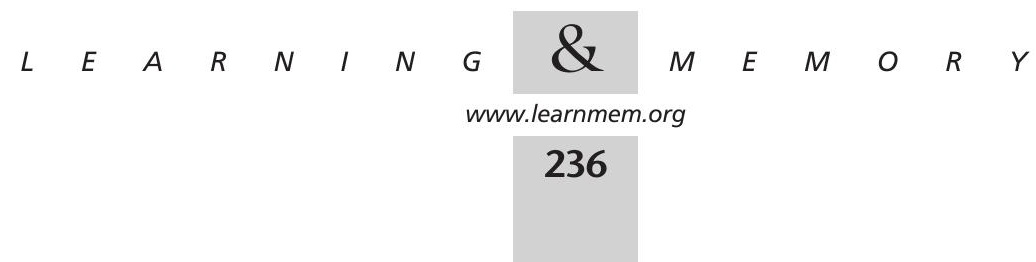




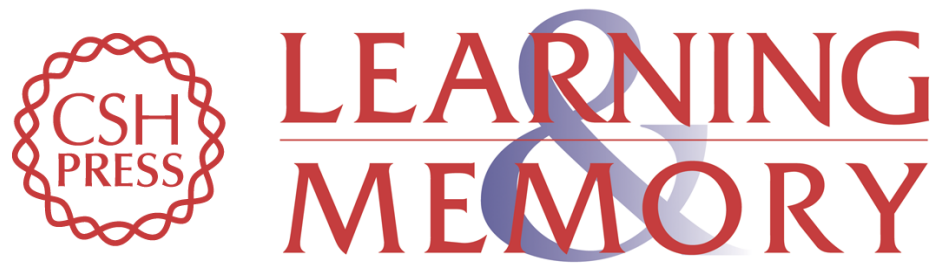

\section{Analysis of Probabilistic Classification Learning in Patients With Parkinson's Disease Before and After Pallidotomy Surgery}

Jennifer R. Sage, Stephan G. Anagnostaras, Shawn Mitchell, et al.

Learn. Mem. 2003, 10:

Access the most recent version at doi:10.1101//m.45903

References This article cites 41 articles, 9 of which can be accessed free at:

http://learnmem.cshlp.org/content/10/3/226.full.html\#ref-list-1

License

Email Alerting Receive free email alerts when new articles cite this article - sign up in the box at the Service top right corner of the article or click here. 